\title{
LOS MODELOS PEDAGÓGICOS Y SU INFLUENCIA EN LA PRACTICA DOCENTE DE LA UNIVERSIDAD FRANCISCO DE PAULA SANTANDER
}

\section{THE TEACHING MODELS AND ITS INFLUENCE ON THE TEACHING PRACTICE, FRANCISCO DE PAULA SANTANDER UNIVERSITY}

\author{
Esp. Duran Chinchilla, Claudia ${ }^{a}$; Msc. Cárdenas García, Maribel ${ }^{\text {b }}$ Ph.D. Velásquez Perez, \\ Torcoroma ${ }^{\mathrm{c}}$
}

${ }^{a}$ Universidad Francisco de Paula Santander Ocaña, Grupo de Investigación GIFEAH, Vía Acolsure Sede el Algodonal, Ocaña - Norte de Santander, Colombia, cmduranc@ufpso.edu.co

${ }^{\mathrm{b}}$ Universidad Francisco de Paula Santander Ocaña, Grupo de Investigación ROTA, Vía Acolsure Sede el Algodonal, Ocaña - Norte de Santander, Colombia, mcardenasg@ufpso.edu.co

${ }^{a}$ Universidad Francisco de Paula Santander Ocaña, Grupo de Investigación GITYD, Vía Acolsure Sede el Algodonal, Ocaña - Norte de Santander, Colombia, tvelaszquep@ufpso.edu.co

Resumen: El proceso investigativo llevado a cabo, tuvo como objetivo primordial determinar cuáles son las prácticas docentes y su relación con el modelo pedagógico de la Universidad Francisco de Paula Santander Ocaña; estuvo enmarcado bajo una investigación cualitativa, toda vez que se tuvo en primera instancia, que acudir a la técnica de la entrevista y grupos focales, como también a la observación, los cuales permitieran reconocer algunas prácticas educativas de los docentes.

El estudio permitió determinar que las prácticas docentes no están alineadas al modelo pedagógico de la Universidad, el cual es el Constructivismo Social, por lo que se hace necesario establecer un plan de mejoramiento orientado a fortalecer las prácticas docentes y que estas a su vez fueran pertinentes con el modelo pedagógico

Palabras clave: modelo pedagógico, Prácticas Educativas, enseñanza, aprendizaje

\begin{abstract}
The research process undertaken, had as its main objective to determine what teaching practices and their relationship with the pedagogical model of Francisco de Paula Santander University Ocaña ; was framed under a qualitative research, since it was in the first instance, to go to the art of the interview and focus groups, as well as the observation, which allowed recognize some educational practices of teachers.
\end{abstract}


The study allowed us to determine which teaching practices are not aligned to the pedagogical model of the University, which is the Social Constructivism, so it is necessary to establish an improvement plan aimed at strengthening teaching practices and that these in turn were relevant with the pedagogical model

Keywords: pedagogical model, Educational Practices, teaching, learning.

\section{INTRODUCCIÓN}

La educación como proceso de socialización, es tan antigua como el mismo ser humano; como toda creación natural ha sido un tema de gran debate. De allí que históricamente muchos estudiosos se han ocupado de reflexionar sobre la misma, pero quizás tal reflexión se intensifica y sistematiza al ritmo del desarrollo científicotécnico.

Al transcurrir los años, se han generado diversas teorías, enfoques, modelos pedagógicos que dan razón a un momento histórico, cultural y social; estos constituyen parámetros específicos relacionados con los procesos de enseñanza- aprendizaje ajustados a las características de las instituciones y del medio social en la cual se desarrollan dichas instituciones.

Es de importancia, en este momento, entender que el que el saber pedagógico no se refiere solo a lo que se ha pensado en torno a la educación, sino que está caracterizado sobre todo por la reflexión que el docente está haciendo en forma constante a partir o con respecto a lo que realiza en el procesos de enseñanza

Desde la perspectiva anterior y teniendo en cuenta que las reflexiones en torno a los procesos pedagógicos, son constantes y diversos, podría decirse que en la actualidad las crisis educativa, no se resuelve eligiendo entre la variada oferta de estrategias y metodologías las más adecuadas, dejando de lado métodos que en este momento son llamados arcaicos, tradicionales, en el sentido que muchos manifiestan que no sirven y que hay que aplicar nuevas formas de enseñanza, afirmación arriesgada, pues quizás no existe procedimiento que sea más adecuado que el otro, mejor que el otro; de tal manera que para estar en lo correcto se tuviera que desechar todo lo que individualmente se considere inadecuado; lo que sí se puede hacer es generar discusión alrededor de este tema trascendental y por supuesto preguntarse: ¿por qué unos métodos suelen ser más adecuados que otros? ¿Por qué se manifiesta abiertamente que la cátedra magistral ya no sirve y que hay que reemplazarla por otras formas de impartir el saber? ¿En qué consiste la transformación curricular?

Como lo establece Murcia, citado por Prieto (2002).

El análisis de la realidad concreta y cotidiana de las salas de clases, permite visualizar la existencia de una combinación de elementos que interactúan entre sí. Los efectos de esta interacción, no sólo a veces apuntan hacia propósitos divergentes, sino que, en muchos casos, aparecen contradictorios respecto de lo que supuestamente deberían ser los objetivos del proceso. La interacción 
implica, necesariamente la comprensión de significados y la interpretación recíproca de los actos propios y en la que la cotidianidad es permanentemente definida y enmarcada en un contexto físico, institucional, histórico y cultural. Estos contextos afectan de manera indiscutible los logros del aprendizaje, por lo que resulta casi ingenuo atribuir relaciones de causalidad entre medios o métodos del profesor y rendimiento, pues son muchos otros los elementos y aspectos que están interactuando e incidiendo en los efectos o resultados del proceso (p.73)

En consecuencia de los aspectos anteriormente citados, surgen los modelos pedagógicos, los cuales según Bernal (2010).

Son representaciones ideales del mundo de lo educativo para explicar teóricamente su hacer. Dichos modelos son dinámicos, se transforman y pueden, en determinado momento, ser aplicados en la práctica pedagógica. Por lo tanto el modelo pedagógico implican los contenidos, los modos de la enseñanza de acuerdo al desarrollo de los niños y niñas y las características de la práctica docente que pretende lograr aprendizajes y se concreta en el aula. Se considera también el modelo pedagógico como un instrumento de la investigación de carácter teórico creado para reproducir idealmente el proceso enseñanza aprendizaje, paradigma que sirve para entender, orientar y dirigir la educación. Se hace evidente la diversidad de conceptos determinantes asociados a la definición de modelo pedagógico (p.4).
Por otro lado de acuerdo a Gago (2002) "un modelo pedagógico, es una representación arquetípica o ejemplar del proceso de enseñanza-aprendizaje, en la que se exhibe la distribución de funciones y la secuencia de operaciones en la forma ideal, que resulta de las experiencias recogidas al ejecutar una teoría del aprendizaje" (p.2)

El estudio de los modelos pedagógicos permite a los docentes tener un panorama de cómo se elaboran los programas, de cómo operan y cuáles son los elementos que desempeñan un papel determinante en un programa o en una planeación didáctica. En algunos de ellos los profesores pueden ver claramente los elementos más generales que intervienen en una planeación didáctica, así como las relaciones de antecedente y consecuente que guardan entre sí. De allí que Porlan (1993) manifiesta:

Para poder identificar un modelo pedagógico se necesita conocer sus características fundamentales que, según Porlan surgen al responder tres preguntas esenciales: ¿Que enseñar? Es decir, qué contenidos, en qué secuencias y en qué orden, su enseñabilidad y relevancia; ¿Cómo enseñar? Se refiere a los métodos, medios y recursos. Aquí adquieren un Valor relevante los estilos de enseñanza de los maestros y de aprendizaje de los estudiantes: ¿Qué y cómo evaluar? Referido no sólo a los momentos, sino también a los instrumentos de comprobación y a los contenidos previstos desde el inicio del proceso. En este aspecto también adquieren importancia los estilos de enseñanza y aprendizaje (p.15).

De allí se hace necesario plantear que todo 
modelo pedagógico debe responder a un enfoque, una metodología y una evaluación; es indispensable conocer también qué percepción tienen de estos tres elementos los estudiantes, los mismos docentes para con ello facilitar y aclarar qué modelo pedagógico puede ser pertinente de acuerdo a las características curriculares, colectivas y sociales, además permite concebir de manera clara la practica educativa.

Según Astolfi, (1997) hay tres modelos predominantes en la enseñanza: "transmitivo, de condicionamiento y constructivista, que sirven -explícita o implícitamente-, como base para las prácticas y estilos de los maestros. Cada uno dispone de una lógica y de una coherencia que habrá de caracterizarlo, pero sobre todo, cada uno responde a diferentes situaciones de eficiencia" (p.34)

Continua diciendo Astolfi et al (1997)

El modelo transmitivo o tradicional se refiere principalmente a la elaboración de un programa de estudios. Los elementos que presentan son mínimos, ya que no se hacen explícitas las necesidades sociales, la intervención de especialistas, las características del educando, ni tampoco se observan las instancias de evaluación del programa de estudios. El esquema es muy sencillo. En él destacan los cuatro elementos siguientes:

- El profesor, que es el elemento principal en el modelo tradicional, ya que tiene un papel activo: ejerce su elocuencia durante la exposición de la clase, maneja numerosos datos, fechas y nombres de los distintos temas, y utiliza el pizarrón de manera constante.

- El método: Se utiliza cotidianamente la clase tipo conferencia, abundantes apuntes, la memorización, y la resolución de los cuestionarios que presentan los libros de texto.

- El alumno, que en este modelo no desempeña una función importante, pues su papel es más bien receptivo, es decir, es tratado como objeto del aprendizaje y no se le da la oportunidad de convertirse en sujeto del mismo.

- La información o contenido se presenta como temas, sin acotar la extensión ni la profundidad con la que deben enseñarse. De esta manera, algunos profesores desarrollan más unos temas que otros creando distintos niveles de aprendizaje en grupos de un mismo grado escolar.

Este Modelo concibe la enseñanza como una actividad artesanal y al profesor como un artesano cuya función es explicar claramente y exponer el conocimiento a los estudiantes de manera progresiva. $\mathrm{Si}$ se presentan errores, son atribuibles al alumno por no adoptar la actitud esperada. Dentro de esta concepción educativa se pueden distinguir dos enfoques de la práctica del docente:

Astolfi et al (1997.p 23) el primero es un enfoque enciclopédico, en el que el profesor es un especialista lleno de información; la enseñanza es una pura transmisión que al final se resume en la acumulación de conocimientos y no se hace distinción entre saber y saber enseñar.

El segundo enfoque es el comprensivo, en el 
que el profesor es un intelectual que comprende lógicamente la estructura de la materia pero sólo la transmite. En ambos enfoques se resta importancia al conocimiento pedagógico que no esté relacionado con las disciplinas en su modo de transmisión y presentación, ni al conocimiento que se deriva de la experiencia práctica como docente. Se trata de un aprendizaje basado en la teoría.

En resumen, en esta perspectiva el aprendizaje es la simple comunicación entre emisor (maestro) y receptor (alumno) y se ignora el fenómeno de comprensión y el proceso de la relación con sentido de los contenidos.

Siguiendo a Astolfi, et al (1997 p.13) el modelo de condicionamiento o pedagogía (conductista), está basado en los estudios de Skinner y Pavlov sobre aprendizaje; se enfatiza en los medios necesarios para llegar a un comportamiento esperado y verificar su obtención.

Finalmente, el Modelo Constructivista o de perspectiva radical, concibe la enseñanza como una actividad crítica, y al docente como a un profesional autónomo que investiga reflexionando sobre su práctica. Este modelo difiere de los anteriores en la forma como se maneja el concepto de error: es un indicador que permite hacer análisis de los procesos intelectuales que ocurren al interior de quien aprende. Para el constructivismo, aprender es arriesgarse a errar (ir de un lado a otro), y muchos de los errores cometidos en situaciones didácticas deben considerarse como momentos creativos. Para el constructivismo la enseñanza no es una simple transmisión de conocimientos; es una tarea de organización de métodos de apoyo y situaciones de aprendizaje que permiten a los alumnos construir su propio saber. No se aprende sólo registrando en el cerebro. Se aprende construyendo la propia estructura cognitiva.

Desde las apreciaciones anteriores, la Educación superior debe garantizar una educación de calidad; para ello es fundamental que los programas que se oferten se fundamenten en currículos que ofrezcan a los estudiantes el desarrollo de competencias que se ajusten a las necesidades de la sociedad.

Indudablemente, uno de los componentes básicos en la calidad educativa, son las prácticas docentes, estas, son variables fundamentales en los procesos académicos, ya que de las buenas prácticas docentes depende la formación de los futuros profesionales, formación que debe estar de la mano con lo que la sociedad exige.

Las prácticas docentes no pueden estar desarticuladas con el modelo pedagógico que las universidades adoptan teniendo en cuenta el contexto, dicho modelo pedagógico debe verse reflejado a través de los métodos, técnicas y metodologías aplicadas a través del desarrollo didáctico de la asignatura, por supuesto no se puede dejar de lado la evaluación, la cual también debe estar correlacionada con el modelo pedagógico; de esa manera, el proceso de enseñanza- aprendizaje y la calidad académica sería más pertinente.

Desde lo anterior, se hizo importante realizar un estudio que permitiera determinar y conocer las prácticas docentes y su relación con el modelo pedagógico de la Universidad, en este caso, con el modelo pedagógico de la Universidad Francisco de 
Paula Santander Ocaña, el cual corresponde al modelo Constructivista Social.

El Proceso investigativo dejó concluir que hace indispensable iniciar un proceso de formación y autoformación por parte de los docentes que ayude a transformar al docente, ya que se consideró que el modelo pedagógico de la Universidad en primera mediada, no es conocido, solo se identifica el nombre, pero, se desconoce las bases epistémicas del mismo, sus características, su didáctica, en fin no hay claridad al respecto, en consecuencia, las clases son una mezcla de distintos modelos.

La formación no es tarea fácil ya que esto exige involucrar una serie de cambios en los diseños curriculares, en el diseño de la institución como eje fundamental del proceso, modos de evaluar la docencia y al docente, por lo que éste proceso implica que la Institución trace un plan de mejoramiento institucional y docente a largo y corto plazo.

De tal manera, pues, lo que hay que emprender es la reflexión pedagógica a través de una forma holística, resultando conveniente no buscar fórmulas de acuerdo a posibles modelos y / $\mathrm{o}$ estrategias metodológicas sino más bien entender lo pedagógico como una toma de decisiones críticas acerca del proceso mismo de la educación convirtiendo al docente, acá, como un eje fundamental; dicho de otra manera, el docente se convierte en un actor principal en el proceso pues es él quien define y articula las distintas metodologías que permiten mejorar los procesos académicos.

Indiscutiblemente, desde la mirada anterior, el saber pedagógico, no puede limitarse a un saber instrumental acerca de cómo manejar y aplicar un modelo curricular adoptado o en vías de ser adoptado e impuesto desde la instancia superior tal como lo exige la actual racionalidad instrumental y funcional, de acuerdo con los lineamientos de la tecno ciencia; estas apertura ha implicado que la modernización exigida se ha entendido como la superación acrítica del pasado, al respecto Bedoya (2011) indica que:

Hay que adoptar necesariamente el nuevo esquema impuesto ya que estas serán las nuevas reglas de juego, por lo tanto deseosos de que todo cambie o de que la corriente de cambio los envuelva se ha cambiado por cambiar, sin entender porque hay que hacerlo, sin asumir el reto de la reflexión (p.45).

Por otro lado y respecto a las anteriores apreciaciones, no se puede dejar de lado y restarle importancia a la llamada "revolución cognitiva", es valioso destacar que en este siglo, la comprensión del aprendizaje se debe ver reflejada en una mayor comprensión de la naturaleza social del aprendizaje, del impacto del contexto en los procesos de pensamientos de los educandos

\section{METODOLOGÍA}

El estudio estuvo fundamentado en la investigación cualitativa, toda vez que esta investigación permite por su propia naturaleza, abordar de manera dialéctica y sistemática el objeto de investigación, de acuerdo a Miguelez (2005) "La investigación cualitativa usa métodos inferenciales con el propósito de elaborar teorías que permitan comprender los 
fenómenos humanos, culturales y sociales" (p.5).

Por su parte, también en un momento de la investigación se acudió a la investigación cuantitativa ya que como lo plantea Valdez (2007) "la investigación cuantitativa, usa métodos deductivos con el propósito de verificar las teorías que explican los hechos reales" (p.21)

De tal manera, no se descartó la aplicación de una encuesta, ya que se necesitaba hacer un balance general en cuanto al conocimiento del modelo pedagógico de la Universidad, en este caso, del Constructivismo Social, en este caso se aplicó el instrumento a toda la población, la cual correspondía a 244 docentes, tiempo completo, medio tiempo y catedráticos. Así mismo, y partiendo de que la investigación es de carácter cualitativo, se acudió a los informantes claves.

En lo que respecta a las técnicas e instrumentos de recolección de información se tuvo en cuenta en primera medida la observación, entrevistas estructuradas y grupos focales; el formato fue sometido a prueba piloto y a la validación de expertos, las preguntas guías fueron: ¿Cómo se ve como docente? ¿Cómo me ven los demás?, ¿soy un docente ideal, porque? ¿Cuáles son los valores que orientan la actuación docente? ¿La acción docente viene de un modelo en el cual fui formado o por el contrario de la persona que soy? ¿Cuál es la formación docente?, entre otras.

Los resultados fueron analizados a través de la triangulación, la cual se llevó a cabo teniendo en cuenta en primera medida que esta permitió los que Hammesersley y Atkinson (1994) llaman.

La comprobación de información referente a un mismo fenómeno obtenida en diferentes fases del trabajo de campo, en diferentes puntos de trabajo de campo, en diferentes puntos o ciclos temporales existentes en el lugar o comparando los relatos de los distintos participantes (p.7)

\section{RESULTADOS}

Como fase inicial, se aplicó una encuesta la cual permitió diagnosticar en primera instancia que el $60 \%$ de los docentes de la Universidad no conoce el modelo pedagógico, un $20 \%$ han escuchado hablar de él pero no saben cómo se desarrolla, un $20 \%$ dicen conocer el modelo y lo aplican en el desarrollo de las clases, esta última apreciación es un poco contradictoria pues al contestar las siguientes preguntas no guardan relación con la Metodología empleada para el desarrollo de las clases.

\section{Conocimiento del modelo pedagógico}

No conocen el modelo pedagógico.

Han escuchado hablar, pero no saben cómo se desarrolla.

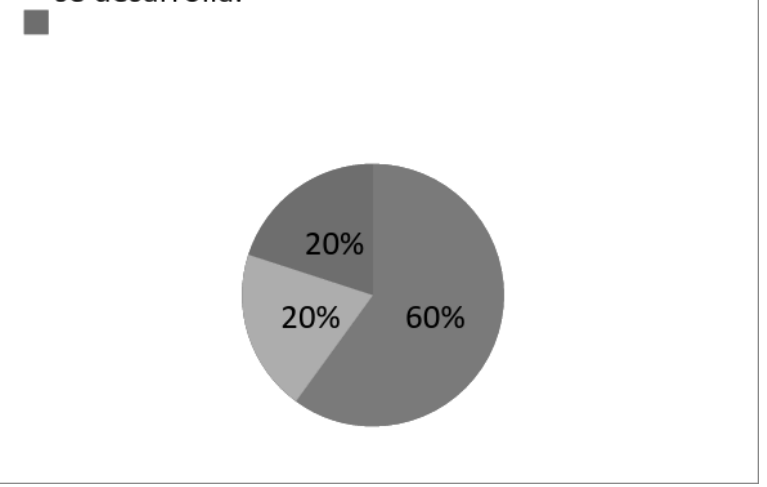

Figura 1. Conocimiento del Modelo Pedagógico.

Fuente: Elaboración propia.

En relación con las técnicas, métodos y recursos utilizados por los docentes en el desarrollo de las clases se puede destacar que en un $58 \%$ de docentes usa el taller, el 
$30 \%$ exposiciones y clase magistral, y un $12 \%$ utiliza técnicas de expresión oral como la mesa redonda, debates, lluvias de ideas.

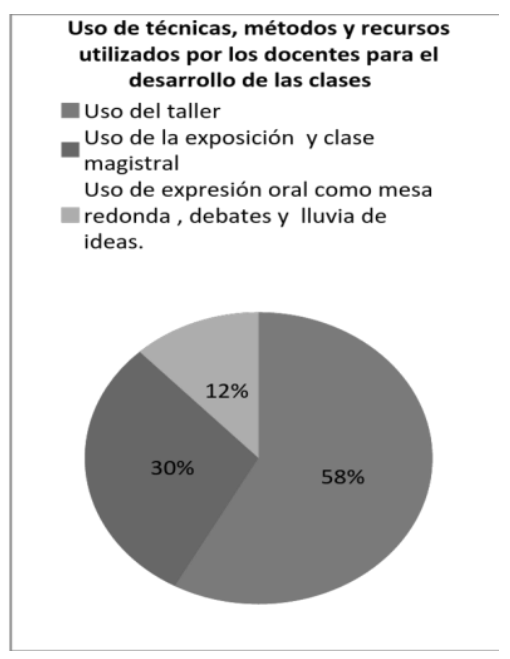

Figura 2. Uso Técnicas y Métodos.

Fuente: Elaboración propia.

Como recurso didáctico se puede establecer que un $25 \%$ de docentes usan algunos recursos didácticos como el videobean $20 \%$ complementan sus cases a través de la plataforma virtual y el $65 \%$ no usa recurso tecnológico.

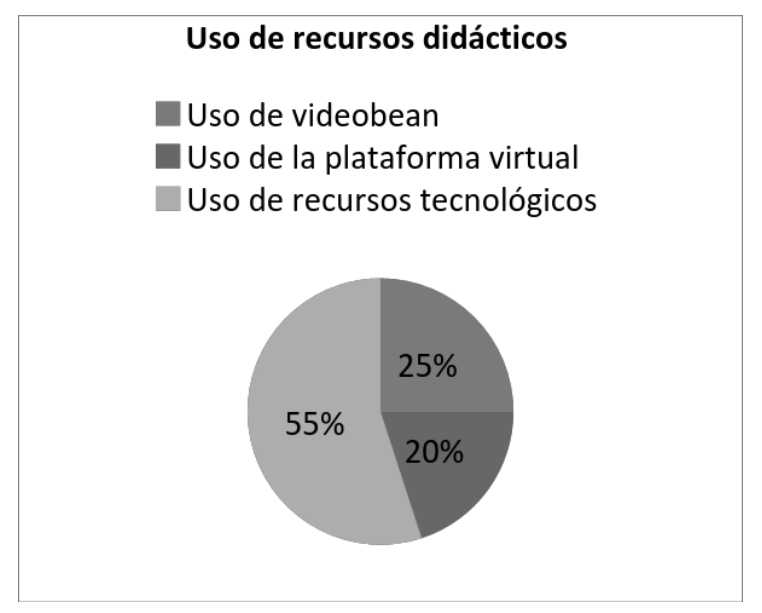

Figura 3. Uso de Recurso didáctico.

Fuente: Elaboración propia.

Los datos obtenidos a través del instrumento permiten hacer las siguientes reflexiones: no hay claridad en lo que respecta al modelo pedagógico propuesto por la Universidad (constructivista Social); por otro lado, no se conoce, no se sabe qué es un modelo pedagógico; cada docente establece su propio modelo. No hay claridad y se tiende a confundir términos como: didáctica, pedagogía, método, metodología, técnica y recursos didácticos, no existe concordancia entre la metodología y el proceso de evaluación y por supuesto el modelo pedagógico que se dice aplicar.

En la siguiente fase del estudio, en la cual se realizaron las entrevistas y se trabajó con los grupos focales, se pudo determinar que hay razones suficientes que justifican la importancia y necesidad de atender a la formación pedagógica inicial y permanente del profesor de educación Universitaria. En primer lugar, el avance de la ciencia, la tecnología y los variados medios de aprendizaje exigen al profesorado una renovación y adaptación constantes a los cambios sociales y educativos. De hecho, el profesor debe poseer un gran dominio de la materia que imparte $y$, a la vez, una actitud permanente de apertura al cambio y la capacidad de ir renovando los contenidos académicos y la metodología de enseñanza a los nuevos conocimientos.

El estudio permite aseverar que la planta docente de la UFPSO tiene formación profesional disciplinar, sin embargo, es de resaltar que un porcentaje pequeño tiene formación en pedagogía, razón por la cual, se puede justificar en cierta medida la no apropiación del ejercicio docente. Algunos docentes han realizado la especialización en prácticas docente Universitaria, ya que es una política de la Universidad formar y capacitar al docente, sin embargo, no se está asumiendo con esfuerzo pues la docencia 
exige voluntad orientada hacia el desarrollo de los procesos de formación integral del estudiante, los procesos de producción, socialización y apropiación crítica del conocimiento y los procesos de servicio a la comunidad; es decir, desde la dinámica del conocer, debe permitir al estudiante captar problemas, plantear correctamente interrogantes, dominar métodos apropiados de conocer y pensar. Como práctica pedagógica, la docencia incorpora los procesos de comunicación, socialización e interacción del y con el conocimiento entre los diferentes actores y agentes del proceso educativo. La docencia es pues, el eje articulador de la investigación y de la proyección social.

Es evidente que hay confusión en lo que respecta a términos básicos del ejercicio docente como: método, técnica, metodología, estrategia y didáctica esta última como tal y como lo establece Escudreo (1981) : "Ciencia que tiene por objeto la organización y orientación de situaciones de enseñanza-aprendizaje de carácter instructivo, tendentes a la formación del individuo en estrecha dependencia de su educación integral" (p. 34). La didáctica entonces, debe reflejar y estar de la mano con la misión y la visión de la universidad, se debe desarrollar de la mano con el modelo pedagógico, modelo que está propuesto desde la mirada del constructivismo social, este modelo expresa una posición epistemológica, en la que explica cómo el ser humano, a lo largo de su vida personal va desarrollando lo que se llama intelecto $\mathrm{y}$ va formando sus conocimientos, el constructivismo basa sus resultados en dos premisas: una que dice que el conocimiento es activamente construido por el sujeto cognoscente, no pasivamente recibido por el entorno; la segunda que plantea que el conocer es un proceso adaptativo que organiza el mundo experiencial de uno. De la misma manera Moreira (1994) dice que: "el Constructivismo no estudia la realidad, sino la construcción de la realidad" (p.3).

En consecuencia, la información suministrada a través del instrumento aplicado en este proceso, permite intuir que el ejercicio docente está encaminado hacia un enfoque tradicional, en donde se evidencia que el docente sigue siendo protagonista del proceso de enseñanza, es depositario del conocimiento y su función es transmitirlo al estudiante, el cual actúa solo como receptor; por otro lado, esta concepción asume que la calidad del docente está en función de la cantidad de conocimiento que se tenga y transmite independientemente del método empleado, esto implica que el docente se desentienda de la responsabilidad del aprendizaje y por lo tanto predominan las estrategias de enseñanza como las exposiciones, talleres y ejercicios o prácticas académicas que el docente cree ser convenientes; el estudiante solo es protagonista de su propio aprendizaje en ciertos momento; contradictorio con lo que el mundo actual está exigiendo en el proceso de formación, el cual debe estar centrado en el aprendizaje. Es decir, las prácticas docentes están siendo desvirtuadas, pues el modelo de la Universidad, como se refleja anteriormente no es asumido, desarrollado, puesto como derrotero en el ejercicio docente, razón por la cual cada uno adopta un modelo diferente de acuerdo a criterios personales y no Institucionales.

En algunos casos existe la tendencia a que los docente intenten ser poco tradicionalistas $\mathrm{y}$ se vuelven un poco progresistas, intentando conducir sus clases a través de la pregunta , aclarando dudas, 
retroalimentando las evaluaciones, realizando trabajos grupales y utilizando algunos recursos didácticos tecnológicos como complemento en su proceso de enseñanza- aprendizaje; es decir se mezclan enfoques convirtiéndose en procesos mixtos.

Teniendo en cuanta las anteriores reflexiones, se hace necesario detenernos a pensar y a repensar el procesos pedagógico, desde dimensiones que coadyuven al mejoramiento del mismo, reflexiones que atiendan a las transformaciones que ha tenido la educación, que permitan entender que cada individuo adopta diferentes conductas; que de paso a concebir que se podría mejorar los procesos académicos siempre y cuando los docentes incorporen prácticas docentes y estas a su vez sean fundamentadas a través de modelos pedagógicos especialmente partiendo del modelo expuesto para la Institución.

Así mismo, y partiendo que los espacios en donde se desarrollan las actividades docentes, especialmente las universidades como centros de formación profesional, generadoras de espacios dinámicos y dialécticos, encargados de transferir y conservar los valores culturales y sociales de las regiones a través de los currículos, deben contribuir al desarrollo y el cambio social y que además contribuya a la formación personal, ética, moral de los individuos. Por ello, los docentes desde el deber ser deben reflexionar sobre sus prácticas docentes para mejorarla y fortalecerla.

De allí, que Diaz (2006), por plantea que:

El saber pedagógico son los conocimientos, construidos de manera formal e informal por los docentes; valores, ideologías, actitudes, prácticas; es decir, creaciones del docente, en un contexto histórico cultural, que son producto de las interacciones personales e institucionales, que evolucionan, se reestructuran, se reconocen y permanecen en la vida del docente (p.21).
Finalmente, es importante reflexionar alrededor de las siguientes preguntas: ¿La universidad ha ofrecido los suficientes espacios de dialogo y debate en torno a los procesos académicos especialmente en lo que respecta al modelo pedagógico? ¿Se ha brindado capacitación permanente a los docentes en lo relacionado al modelo pedagógico?

\section{CONCLUSIONES}

En esta fase inicial, se pudo determinar que para que cualquier modelo pedagógico funcione, es fundamental que las diferentes prácticas docentes estén en concordancia con el mismo, dado que de éstas en gran parte depende la mejora de los procesos educativos; sobre todo en lo que concierne a los nuevos paradigmas de la educación y los nuevos enfoques pedagógicos constructivistas, que este caso es el modelo constructivista social, adoptado por la Universidad Francisco de Paula Santander Ocaña.

Indudablemente, las prácticas docentes son las que inciden en las aptitudes y actitudes, habilidades y destrezas que los estudiantes desarrollen y les permite aprender significativamente no para hoy, sino para la vida profesional y personal; por lo que las estrategias educativas deben estar ajustadas a los estilos de aprendizaje y a los marcos de reconceptualización que la educación y el contexto de hoy exige.

Se hace indispensable pues iniciar un proceso de formación y autoformación por parte de los docentes que ayude a transformarlos, lo cual no es fácil, ya que esto exige involucrar una serie de cambios en los diseños curriculares, en el diseño de la institución como eje fundamental del 
proceso, modos de evaluar la docencia y al docente, por lo que este proceso implica que la Institución trace un plan de mejoramiento institucional y docente a largo y corto plazo.

De igual manera, es importante que el docente realice un autoexamen en cuanto a los siguientes criterios: Formación personal, teórica, disciplinar, investigativa, promulgación de valores, para con ello establecer de qué forma ha sido su formación, y por ende la que él imparte a sus estudiantes: si es artesanal donde un aprendizaje servía para toda la vida; personalista la cual centra la atención sobre la persona con todos sus condicionantes y posibilidades, es decir, la formación deja de ser un proceso de enseñar, para pasar al autodescubrimiento personal, el tomar conciencia de sí mismo, se trata del desarrollo total de la personalidad y no de conductas específicas; academicista el cual tiene como objetivo fundamental de formación la trasferencia de conocimientos científicos y culturales; técnica en la cual el docente domina las aplicaciones del conocimiento científico producido por otros y luego las aplica; o si bien en de orientación práctica la fundamentación es que la enseñanza actividad compleja se desarrolla en escenarios singulares, claramente determinada por el contexto; orientación social-reconstruccionista, que pone de relieve el carácter crítico de la enseñanza entendida como una actividad social saturada de opciones de carácter ético, en la que los valores que presiden su intencionalidad deben traducirse en principios de procedimiento que rijan y se realicen a lo largo de todos los procesos de enseñanza y aprendizaje.

Es posible, entonces, que la formación docente esté mediada de manera evidente por uno de los modelos anunciados anteriormente; o que tenga elementos de uno u otro, queda entonces para la reflexión personal determinar cómo puedo identificar el modelo de formación docente para con ello relacionarlo con el modelo establecido por la Universidad y de esta manera alinearse con el mismo, pues fundamentar que exista articulación entre las prácticas pedagógicas y los modelos pedagógicos Universitarios, que en este caso particular el modelo de la Universidad Francisco de Paula Santander Ocaña.

\section{FINANCIACIÓN}

- Las autoras del proyecto, agradecen en primera instancia a la Universidad por abrir espacios en los que se le permite a los docentes realizar proyectos de investigación que de una $u$ otra manera aportan al fortalecimiento de los procesos académicos y pedagógicos.

- En segunda instancia se agradece a los docentes que aportaron sus experiencias en la recolección de información.

- A la DIE por generar escenario investigativos y apoyar los procesos.

\section{BIBLIOGRAFÍA}

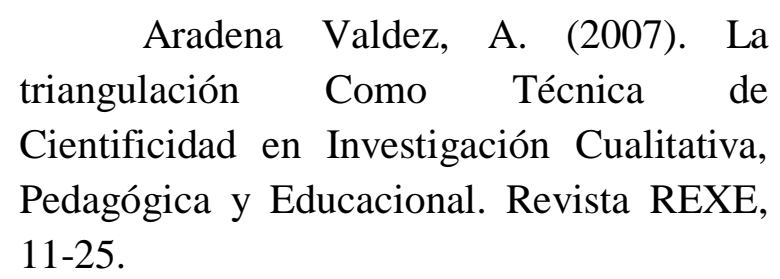

Astolfi, J. (1997). Aprender en la Escuela. Chile: Dolmen.

Bedoya, J. (2011). Pedagogía ¿Enseñar a Pensar? Bogotá: Ecoe Ediciones. 
Diaz Cuero, V. (2006). Formación Docente, Práctica Pedagógica y Saber Pedagógico Volumen 12. Luarus, revista de Educación, 88-103.

Escudero, J. (1981). Modelos Didácticos. Barcelona: Oikus-tao.

Gago, H. (2002). Apuntes Sobre la Evaluación Educativa. México: Secretaria de Educación Pública.

Hammersley, M., \& Atkinson, P. (1994). Etnografía. Métodos de Investigación. Barcelona: Paidos.

Martínez Miguelez, M. (2005). Actualización de la Epistemología y Metodología en Educación. III jornada de Innovación Educativa (págs. 1-5). Barquisimeto: Lara.

Moreira, M. (1994). Diez Años de la Revista de la Enseñanza de Las Ciencias: de Una Ilusión a Una Realidad.

Pino Bernal, L. (2010). Análisis Comparativo de los Modelos Pedagógicos Aplicados en el décimo año de Educación Básica del Centro Educativo Particular "Espíritu Santo". Guayaquil: Universidad Católica De Guayaquil.

Porlan, R. (1993). Constructivismo y Escuela. Hacia Un Modelo De Enseñanza Aprendizaje, basado en Investigación. España: Diada.

Prieto Parra, M. (2002). La práctica Pedagógica en el Aula: Un análisis Crítico. Revista Educación y Pedagógica, 73 - 85. 